\title{
EQUIVALENCE CLASS IN THE SET OF FUZZY NUMBERS AND ITS APPLICATION IN DECISION-MAKING PROBLEMS
}

\author{
GEETANJALI PANDA, MOTILAL PANIGRAHI, AND SUDARSAN NANDA
}

Received 4 November 2005; Revised 20 June 2006; Accepted 22 June 2006

An equivalence relation is defined in the set of fuzzy numbers. In a particular equivalence class, arithmetic operations of fuzzy numbers are introduced. A fuzzy matrix with respect to a particular class and its associated crisp matrices are also introduced. The concept of equivalence class is applied in fuzzy decision-making problems and justified through a numerical example.

Copyright (c) 2006 Hindawi Publishing Corporation. All rights reserved.

\section{Introduction}

Since the discovery of fuzzy sets, the arithmetic operations of fuzzy numbers (Zadeh $[7,8]$ ) which may be viewed as a generalization of interval arithmetic (Moore [5]) have emerged as an important area of research within the theory of fuzzy sets (Mizumoto and Tanaka [4], Dubois and Prade [2]). The arithmetic operations of fuzzy numbers have been performed either by extension principle $[7,8]$ or by using alpha cuts as discussed by Dubois and Prade [2]. If two triangular (linear) fuzzy numbers are added or subtracted by applying extension principle, then the result is again a triangular fuzzy number. However, when we take other operations like multiplication or division, then the result is not a linear triangular fuzzy number. Thus these operations are not closed in the sense that operations of two same types (triangular/trapezoidal, etc.) of fuzzy numbers may not necessarily result in fuzzy number of that type (triangular/trapezoidal, etc.). Hence it is cumbersome to find the membership function of the arithmetical operations of large number of fuzzy numbers (may be of same type) by these principles. In the present paper, an attempt is made to overcome such type of difficulties. In Section 3, a relation between two fuzzy numbers is defined and it has been proved that this relation divides the whole set of fuzzy numbers into equivalence classes. In Section 4, arithmetic operations in a particular class are defined. These operations are different from the arithmetic operations of fuzzy numbers, developed by extension principle or alpha cut. It has been proved and also verified through examples that these arithmetic operations are closed in their respective 
classes (e.g., product of two triangular fuzzy numbers in a particular class results in a triangular fuzzy number of the same class).

$[\mu]$-fuzzy matrix and arithmetic operations of $[\mu]$-fuzzy matrices are introduced in Section 5. Some properties such as definiteness, symmetry of $[\mu]$-fuzzy matrices (these are useful to formulate quadratic programming) are discussed.

To make these concepts of equivalence class, arithmetic operations in a particular class and $[\mu]$-fuzzy matrix useful in practical sense, an attempt is made to apply it in fuzzy decision-making problems. Fuzzy decision making was discovered by Bellman and Zadeh [1] and a lot of work has been done by many researchers like Zimmermann [9], Werners [6], and so forth to solve fuzzy decision-making problems using different types of defuzzification methods. In this paper, a different type of solution method for fuzzy decision-making problem has been developed without defuzzifying it. In any defuzzification method, solution of a fuzzy decision-making problem does not involve the aspiration level of the fuzzy numbers present in the problem. But in our solution method, each aspiration level corresponds to a solution. We have considered a fuzzy decision-making problem whose coefficients are fuzzy numbers belonging to a particular class. A general fuzzy decision-making problem in matrix form is formulated and its solution method is given in Section 6. The methodology is verified for fuzzy quadratic programming problem.

Throughout this paper, we have considered the set of all fuzzy numbers with compact support and also LR type.

\section{Some preliminary results on fuzzy numbers}

Definition 2.1 (fuzzy number). A fuzzy number is a fuzzy subset of the real line $\mathbb{R}$ which is convex and normal.

Let $F$ be the set of all fuzzy numbers which are upper semicontinuous and have compact support. The support of a fuzzy number $\tilde{a}$ with membership function $\mu_{\tilde{a}}: \mathbb{R} \rightarrow[0,1]$ is denoted by $\operatorname{supp}(\tilde{a})$, where $\operatorname{supp}(\tilde{a})=\left[l_{a}, r_{a}\right]$ and

$$
\mu_{\widetilde{a}}(t)= \begin{cases}\mu_{\tilde{a} L}(t), & l_{a} \leq t \leq a_{*}, \\ 1, & a_{*} \leq t \leq a^{*}, \\ \mu_{\widetilde{a} R}(t), & a^{*} \leq t \leq r_{a}, \\ 0, & \text { otherwise. }\end{cases}
$$

$\mu_{\tilde{a} L}:\left[l_{a}, a_{*}\right] \rightarrow[0,1]$ is a continuous and strictly increasing function and $\mu_{\tilde{a} R}:\left[a^{*}, r_{a}\right] \rightarrow$ $[0,1]$ is a continuous and strictly decreasing function. $\mu_{\tilde{a}}(t)=1$ for $t \in\left[a_{*}, a^{*}\right]$ and is called the core of $\tilde{a}$, denoted by core $(\tilde{a})$. Symbolically, the fuzzy number $\tilde{a}$ with compact support is represented by $\tilde{a}=\left\langle l_{a} ; a_{*} ; a^{*} ; r_{a}\right\rangle$.

Definition 2.2 (triangular fuzzy number). A fuzzy number $\tilde{a}=\left\langle l_{a} ; a_{*} ; a^{*} ; r_{a}\right\rangle$ is said to be a triangular fuzzy number if $a_{*}=a^{*}$. Moreover, if the membership function $\mu_{\tilde{a}}$ is such that $\mu_{\tilde{a} L}$ and $\mu_{\tilde{a} R}$ are linear, then $\tilde{a}$ is a linear triangular fuzzy number. 
Definition 2.3 (LR fuzzy number). A fuzzy number $\tilde{a}$ is of LR type if there exist reference functions $L$ and $R$, scalars $\alpha>0, \beta>0$ with

$$
\mu_{\tilde{a}}(t)= \begin{cases}L\left(\frac{a_{*}-t}{\alpha}\right), & t \leq a_{*}, \\ 1, & a_{*} \leq t \leq a^{*}, \\ R\left(\frac{t-a^{*}}{\beta}\right), & t \geq a^{*} .\end{cases}
$$

$\left[a_{*}, a^{*}\right]$ is the core of $\tilde{a}, \alpha$ and $\beta$ are the left and right spreads, respectively. $L, R: \mathbb{R}^{+} \rightarrow$ $[0,1]$ are decreasing shape functions. $L(0)=R(0)=1, L(1)=R(1)=0($ or $L(t)>0, R(t)>$ 0 , for all $t, L(-\infty)=R(+\infty)=0)$. An LR-type fuzzy number $\tilde{a}$ is represented by $\tilde{a}=$ $\left\langle a_{*} ; a^{*} ; \alpha, \beta\right\rangle_{\mathrm{LR}}$. Let $F^{*}$ denote the set of all LR fuzzy numbers.

Definition 2.4 (ranking of fuzzy numbers [3]). For $0 \leq \lambda \leq 1$, the $\lambda$-integral value of a fuzzy number $\tilde{a}$, denoted by $I_{\lambda}(\tilde{a})$, is

$$
I_{\lambda}(\tilde{a})=\lambda R(\tilde{a})+(1-\lambda) L(\tilde{a})
$$

where

$$
\begin{aligned}
& R(\tilde{a})=\int_{0}^{1} \mu_{\tilde{a} R}^{-1}(\alpha) d \alpha, \\
& L(\tilde{a})=\int_{0}^{1} \mu_{\tilde{a} L}^{-1}(\alpha) d \alpha .
\end{aligned}
$$

For two fuzzy numbers $\tilde{a}$ and $\tilde{b}, \tilde{a} \preceq \tilde{b}$ (less than or equal to in fuzzy sense) if and only if $I_{\lambda}(\tilde{a}) \leq I_{\lambda}(\tilde{b})$ for $0 \leq \lambda \leq 1$.

The next section is devoted to define equivalence class in the set of fuzzy numbers.

\section{Equivalence class in fuzzy numbers}

Before introducing an equivalence relation, we define the domain of a fuzzy number in $F$ and $F^{*}$ as follows.

(I) Suppose $\tilde{a}=\left\langle l_{a} ; a_{*} ; a^{*} ; r_{a}\right\rangle$ and $\tilde{b}=\left\langle l_{b} ; b_{*} ; b^{*} ; r_{b}\right\rangle$ are two fuzzy numbers in $F$ with membership functions $\mu_{\tilde{a}}$ and $\mu_{\tilde{b}}$, respectively. The domains of $\tilde{a}$ and $\tilde{b}$ are $D_{\tilde{a}}$ and $D_{\tilde{b}}$, respectively, which are real lines and can be represented by

$$
\begin{aligned}
& D_{\tilde{a}}=\left(-\infty, l_{a}\right) \cup\left[l_{a}, r_{a}\right] \cup\left(r_{a}, \infty\right), \\
& D_{\tilde{b}}=\left(-\infty, l_{b}\right) \cup\left[l_{b}, r_{b}\right] \cup\left(r_{b}, \infty\right) .
\end{aligned}
$$


4 Equivalence class in fuzzy numbers

Let $f: D_{\tilde{a}} \rightarrow D_{\tilde{b}}$ be defined as

$$
f(t)= \begin{cases}t+l_{b}-l_{a}, & t \leq l_{a}, \\ \frac{b_{*}\left(t-l_{a}\right)+l_{b}\left(a_{*}-t\right)}{a_{*}-l_{a}}, & l_{a} \leq t \leq a_{*}, \\ \frac{b^{*}\left(t-a_{*}\right)+b_{*}\left(a^{*}-t\right)}{a^{*}-a_{*}}, & a_{*} \leq t \leq a^{*}, \\ \frac{r_{b}\left(t-a^{*}\right)+b^{*}\left(r_{a}-t\right)}{r_{a}-a^{*}}, & a^{*} \leq t \leq r_{a}, \\ t+r_{b}-r_{a}, & t \geq r_{a} .\end{cases}
$$

(II) Suppose $\tilde{a}$ and $\tilde{b}$ are in $F^{*}, \tilde{a}=\left\langle a_{*} ; a^{*} ; \alpha, \beta\right\rangle_{\mathrm{LR}}$, and $\tilde{b}=\left\langle b_{*} ; b^{*} ; \alpha, \beta\right\rangle_{\mathrm{LR}}$. Then

$$
\begin{aligned}
& D_{\widetilde{a}}=\left(-\infty, a_{*}-\alpha\right) \cup\left[a_{*}-\alpha, a^{*}+\beta\right] \cup\left(a^{*}+\beta, \infty\right), \\
& D_{\widetilde{b}}=\left(-\infty, b_{*}-\alpha\right) \cup\left[b_{*}-\alpha, b^{*}+\beta\right] \cup\left(b^{*}+\beta, \infty\right) .
\end{aligned}
$$

In this case, $f: D_{\widetilde{a}} \rightarrow D_{\widetilde{b}}$ can be defined as in (3.2), just by replacing $l_{a}$ by $a_{*}-\alpha, l_{b}$ by $b_{*}-\alpha, r_{a}$ by $a^{*}+\beta$, and $r_{b}$ by $b^{*}+\beta$.

It is not hard to see that $f$ is bijective in both cases.

Definition 3.1. For $\tilde{a}, \tilde{b} \in F$ (or $\left.F^{*}\right)$, define a relation $\cong$ between $\tilde{a}$ and $\tilde{b}(\tilde{b}$ is said to be related to $\tilde{a}$ by the relation $\cong$ ) as

$$
\tilde{a} \cong \tilde{b} \quad \text { iff } \mu_{\tilde{a}}(t)=\mu_{\tilde{b}}(f(t))
$$

Example 3.2. Let $\tilde{a}\langle 1 ; 2 ; 4\rangle, \tilde{b}\langle 2 ; 5 ; 7\rangle \in F$ be two linear triangular fuzzy numbers. Then

$$
\begin{gathered}
\mu_{\tilde{a}}(t)=\left\{\begin{array}{ll}
t-1, & 1 \leq t \leq 2, \\
2-\frac{t}{2}, & 2 \leq t \leq 4, \\
0, & \text { otherwise, }
\end{array} \mu_{\widetilde{b}}(t)= \begin{cases}\frac{t-2}{3}, & 2 \leq t \leq 5, \\
\frac{7-t}{2}, & 5 \leq t \leq 7, \\
0, & \text { otherwise, }\end{cases} \right. \\
f(t)= \begin{cases}t+1, & t \leq 1, \\
3 t-1, & 1 \leq t \leq 2, \\
t+3, & t \geq 2 .\end{cases}
\end{gathered}
$$

For all $t, \mu_{\tilde{a}}(t)=\mu_{\tilde{b}}(f(t))$. Hence $\tilde{a} \cong \tilde{b}$. 
Example 3.3. $\tilde{a}=\langle 2 ; 1,2\rangle_{L^{*} R^{*}}, \tilde{b}=\langle 5 ; 1,2\rangle_{L^{*} R^{*}}$ are two fuzzy numbers in $F^{*} . L^{*}(t)=$ $\left(1+t^{2}\right)^{-1}, R^{*}(t)=e^{-t}$. Then

$$
\begin{aligned}
& \mu_{\tilde{a}}(t)= \begin{cases}\frac{1}{1+(2-t)^{2}}, & t \leq 2, \\
e^{-(t-2) / 2}, & t \geq 2,\end{cases} \\
& \mu_{\tilde{b}}(t)= \begin{cases}\frac{1}{1+(5-t)^{2}}, & t \leq 5, \\
e^{-(t-5) / 2}, & t \geq 5,\end{cases}
\end{aligned}
$$

$f(t)=t+3$ and $\mu_{\tilde{a}}(t)=\mu_{\tilde{b}}(t+3)$. Hence $\tilde{a} \cong \tilde{b}$.

THEOREM 3.4. $\cong$ is an equivalence relation in $F$.

Proof. Let $\tilde{a}, \tilde{b}, \tilde{c} \in F, \tilde{a}=\left\langle l_{a} ; a_{*} ; a^{*} ; r_{a}\right\rangle, \tilde{b}=\left\langle l_{b} ; b_{*} ; b^{*} ; r_{b}\right\rangle$, and $\tilde{c}=\left\langle l_{c} ; c_{*} ; c^{*} ; r_{c}\right\rangle$.

(i) Obviously, $\cong$ is reflexive.

(ii) Let $\tilde{a} \cong \widetilde{b}$, that is, $\mu_{\widetilde{a}}(t)=\mu_{\tilde{b}}(f(t))$, where $f$ is defined as in (3.2). Since $f$ is bijective, $f^{-1}: D_{\tilde{b}} \rightarrow D_{\tilde{a}}$ exists and is defined as

$$
f^{-1}(t)= \begin{cases}t+l_{a}-l_{b}, & t \leq l_{b}, \\ \frac{a_{*}\left(t-l_{b}\right)+l_{a}\left(b_{*}-t\right)}{b_{*}-l_{b}}, & l_{b} \leq t \leq b_{*}, \\ \frac{a^{*}\left(t-b_{*}\right)+a_{*}\left(b^{*}-t\right)}{b^{*}-b_{*}}, & b_{*} \leq t \leq b^{*}, \\ t+r_{a}-r_{b}, & b^{*} \leq t \leq r_{b}, \\ r_{b}-b^{*}\left(t-b^{*}\right)+a^{*}\left(r_{b}-t\right) & t \geq r_{b} .\end{cases}
$$

It is easy to see that $f^{-1}(f(t))=f f^{-1}(t)=t$ for each $t$. Also

$$
\mu_{\tilde{a}}\left(f^{-1}(t)\right)= \begin{cases}\mu_{\tilde{a} L}\left(f^{-1}(t)\right), & l_{a} \leq f^{-1}(t) \leq a_{*}, \\ 1, & a_{*} \leq a^{*}, \\ \mu_{\tilde{a} R}\left(f^{-1}(t)\right), & a^{*} \leq f^{-1}(t) \leq r_{a}, \\ 0, & \text { otherwise }\end{cases}
$$


6 Equivalence class in fuzzy numbers

$$
\begin{aligned}
& = \begin{cases}\mu_{\tilde{b} L}\left[\frac{b_{*}\left(f^{-1}(t)-l_{a}\right)+l_{b}\left(a_{*}-f^{-1}(t)\right)}{a_{*}-l_{a}}\right], & l_{a} \leq f^{-1}(t) \leq a_{*}, \\
1, & a_{*} \leq f^{-1}(t) \leq a^{*}, \\
\mu_{\tilde{b} R}\left[\frac{r_{b}\left(f^{-1}(t)-a^{*}\right)+b^{*}\left(r_{a}-f^{-1}(t)\right)}{r_{a}-a^{*}}\right], & a^{*} \leq f^{-1}(t) \leq r_{a}, \\
0, & \text { otherwise }\end{cases} \\
& = \begin{cases}\mu_{\tilde{b} L}(t), & l_{b} \leq t \leq b_{*}, \\
1, & b_{*} \leq t \leq b^{*}, \\
\mu_{\tilde{b} R}(t), & b^{*} \leq t \leq r_{b}, \\
0, & \text { otherwise }\end{cases} \\
& =\mu_{\tilde{b}}(t) .
\end{aligned}
$$

Hence $\tilde{b} \cong \tilde{a}$, that is, $\cong$ is symmetric.

(iii) Let $\tilde{a} \cong \tilde{b}$ and $\tilde{b} \cong \widetilde{c}$. $f: D_{\widetilde{a}} \rightarrow D_{\widetilde{b}}$ is as defined in (3.2) and $g: D_{\tilde{b}} \rightarrow D_{\widetilde{c}}$ is

$$
g(t)= \begin{cases}t+l_{c}-l_{b}, & t \leq l_{b}, \\ \frac{c_{*}\left(t-l_{b}\right)+l_{c}\left(b_{*}-t\right)}{b_{*}-l_{b}}, & l_{b} \leq t \leq b_{*}, \\ \frac{c^{*}\left(t-b_{*}\right)+c_{*}\left(b^{*}-t\right)}{b^{*}-b_{*}}, & b_{*} \leq t \leq b^{*}, \\ \frac{r_{c}\left(t-b^{*}\right)+c^{*}\left(r_{b}-t\right)}{r_{b}-b^{*}}, & b^{*} \leq t \leq r_{b}, \\ t+r_{c}-r_{b}, & t \geq r_{b},\end{cases}
$$

such that $\mu_{\tilde{a}}(t)=\mu_{\tilde{b}}(f(t)), \mu_{\tilde{b}}(t)=\mu_{\tilde{c}}(g(t))$. So $\mu_{\tilde{a}}(t)=\mu_{\tilde{c}}(g(f(t)))=\mu_{\tilde{c}}\left(\left(g_{o} f\right)(t)\right)$, where $g_{o} f: D_{\tilde{a}} \rightarrow D_{\tilde{c}}$ is

$$
g_{o} f(t)= \begin{cases}\frac{f(t)+l_{c}-l_{b},}{\frac{c_{*}\left(f(t)-l_{b}\right)+l_{c}\left(b_{*}-f(t)\right)}{b_{*}-l_{b}},} & f(t) \leq l_{b}, \\ c^{*}\left(f(t)-b_{*}\right)+c_{*}\left(b^{*}-f(t)\right) & l_{b} \leq f(t) \leq b_{*}, \\ \frac{r_{c}\left(f(t)-b^{*}\right)+c^{*}\left(r_{b}-f(t)\right)}{r_{b}-b^{*}}, & b_{*} \leq f(t) \leq b^{*}, \\ f(t)+r_{c}-r_{b}, & b^{*} \leq f(t) \leq r_{b}, \\ & f(t) \geq r_{b},\end{cases}
$$




$$
= \begin{cases}t+l_{c}-l_{a}, & t \leq l_{a}, \\ \frac{c_{*}\left(t-l_{a}\right)+l_{c}\left(a_{*}-t\right)}{a_{*}-l_{a}}, & l_{a} \leq t \leq a_{*}, \\ \frac{c^{*}\left(t-a_{*}\right)+c_{*}\left(a^{*}-t\right)}{a^{*}-a_{*}}, & a_{*} \leq t \leq a^{*}, \\ \frac{r_{c}\left(t-a^{*}\right)+c^{*}\left(r_{a}-t\right)}{r_{a}-a^{*}}, & a^{*} \leq t \leq r_{a}, \\ t+r_{c}-r_{a}, & t \geq r_{a} .\end{cases}
$$

Hence $\tilde{a} \cong \tilde{c}$, that is, $\cong$ is transitive.

So $\cong$ is an equivalence relation in $F$.

THEOREM 3.5. $\cong$ is an equivalence relation in $F^{*}$.

Proof. Proof of this result is similar to the proof of Theorem 3.4.

The set of all the fuzzy numbers in $F$ (or $F^{*}$ ), which are equivalent to each other by the relation $\cong$, is known as an equivalence class and we denote it by $[\mu]$. (In fact, the equivalence class $[\mu]$ contains all the fuzzy numbers with similar membership function $\mu$.) Thus $[\mu]$ may be a triangular class, trapezoidal class, Gaussian class or a particular type of LR class, and so forth.

\section{Arithmetic operations of fuzzy numbers in an equivalence class}

Suppose $\tilde{a}, \tilde{b} \in[\mu], \tilde{a}=\left\langle l_{a} ; a_{*} ; a^{*} ; r_{a}\right\rangle$, and $\tilde{b}=\left\langle l_{b} ; b_{*} ; b^{*} ; r_{b}\right\rangle \in[\mu]$,

$$
\begin{aligned}
\operatorname{supp}(\tilde{a}) & =\left[l_{a}, r_{a}\right], \quad \operatorname{core}(\tilde{a})=\left[a_{*}, a^{*}\right], \\
\operatorname{domain~of~} \tilde{a} & =D_{\tilde{a}}=\left(-\infty, l_{a}\right] \cup\left[l_{a}, r_{a}\right] \cup\left[r_{a}, \infty\right), \\
\operatorname{supp}(\tilde{b}) & =\left[l_{b}, r_{b}\right], \quad \operatorname{core}(\tilde{b})=\left[b_{*}, b^{*}\right], \\
\operatorname{domain~of~} \tilde{b} & =D_{\tilde{b}}=\left(-\infty, l_{b}\right] \cup\left[l_{b}, r_{b}\right] \cup\left[r_{b}, \infty\right) .
\end{aligned}
$$

We now define the arithmetic operation $*$ as follows:

$$
\tilde{a} * \tilde{b}=\tilde{c}
$$

where the fuzzy number $\tilde{c}$ is defined as below:

$$
\begin{gathered}
\operatorname{supp}(\tilde{c})=\operatorname{supp}(\tilde{a}) *^{\prime} \operatorname{supp}(\tilde{b})=\left[l_{a}, r_{a}\right] *^{\prime}\left[l_{b}, r_{b}\right]=\left[l_{c}, r_{c}\right] \\
\operatorname{core}(\tilde{c})=\operatorname{core}(\tilde{a}) *^{\prime} \operatorname{core}(\tilde{b})=\left[a_{*}, a^{*}\right] *^{\prime}\left[b_{*}, b^{*}\right]=\left[c_{*}, c^{*}\right] .
\end{gathered}
$$

Here $*^{\prime}$ denotes the arithmetic operation of intervals corresponding to fuzzy arithmetic operation “ $*$ " (i.e., if $*$ represents fuzzy multiplication, then $*^{\prime}$ represents multiplication 
8 Equivalence class in fuzzy numbers

of intervals). The domain of $\tilde{c}$ denoted by $D_{\tilde{c}}$ or $D_{\widetilde{a} * \tilde{b}}$ is

$$
D_{\tilde{c}}=\left(-\infty, l_{c}\right) \cup\left[l_{c}, r_{c}\right] \cup\left(r_{c}, \infty\right) .
$$

Theorem 4.1. If $\tilde{a}, \tilde{b} \in[\mu]$, then $\tilde{a} * \tilde{b} \in[\mu]$.

Proof. Let $\tilde{a}, \tilde{b} \in[\mu]$ and $\tilde{a} * \tilde{b}=\tilde{c}$ is defined as above.

We define $g: D_{\tilde{a}} \rightarrow D_{\widetilde{a} * \tilde{b}}$ by

$$
g(t)= \begin{cases}t+l_{c}-l_{a}, & t \leq l_{a}, \\ \frac{c_{*}\left(t-l_{a}\right)+l_{c}\left(a_{*}-t\right)}{a_{*}-l_{a}}, & l_{a} \leq t \leq a_{*}, \\ \frac{c^{*}\left(t-a_{*}\right)+c_{*}\left(a^{*}-t\right)}{a^{*}-a_{*}}, & a_{*} \leq t \leq a^{*}, \\ \frac{r_{c}\left(t-a^{*}\right)+c^{*}\left(r_{a}-t\right)}{r_{a}-a^{*}}, & a^{*} \leq t \leq r_{a}, \\ t+r_{c}-r_{a}, & t \geq r_{a} .\end{cases}
$$

The membership value of the fuzzy number $\tilde{a} * \tilde{b}$ is defined as

$$
\mu_{\widetilde{a} * \tilde{b}}(g(t))=\mu_{\widetilde{a}}(t) .
$$

Since $g$ is a bijective mapping, so

$$
\mu_{\tilde{c}}(t)=\mu_{\widetilde{a} * \tilde{b}}(t)=\mu_{\tilde{a}}\left(g^{-1}(t)\right) .
$$

Hence $\tilde{a} \cong \tilde{a} * \tilde{b}$, that is, $\tilde{a} * \tilde{b} \in[\mu]$. This proves that $*$ is closed in $[\mu]$.

Let us consider an example of fuzzy multiplication. We denote the multiplication of two fuzzy numbers by $\otimes$.

Example 4.2 (multiplication). Consider the following two fuzzy numbers: $\tilde{a}=\langle 1 ; 3 ; 6 ; 7\rangle$, $\tilde{b}=\langle 2 ; 3 ; 5 ; 8\rangle \in[\mu] .[\mu]$ is a linear trapezoidal class. Then the membership functions of $\tilde{a}$ and $\tilde{b}$ are

$$
\mu_{\tilde{a}}(t)=\left\{\begin{array}{ll}
\frac{t-1}{2}, & 1 \leq t \leq 3, \\
1, & 3 \leq t \leq 6, \\
7-t, & 6 \leq t \leq 7, \\
0, & \text { otherwise }
\end{array} \quad \mu_{\widetilde{b}}(t)= \begin{cases}t-2, & 2 \leq t \leq 3 \\
1, & 3 \leq t \leq 5 \\
\frac{8-t}{3}, & 5 \leq t \leq 8 \\
0, & \text { otherwise }\end{cases}\right.
$$


$\operatorname{supp}(\tilde{a} \otimes \tilde{b})=[2,56], \operatorname{core}(\tilde{a} \otimes \tilde{b})=[9,30]$. Now

$$
g(t)= \begin{cases}t+1, & t \leq 1 \\ \frac{7 t-3}{2}, & 1 \leq t \leq 3 \\ 7 t-12, & 3 \leq t \leq 6 \\ 26 t-126, & 6 \leq t \leq 7 \\ t+49, & t \geq 7\end{cases}
$$

We will evaluate $\mu_{\tilde{a} * \tilde{b}}$ as below.

(i) $t \leq 2 \Rightarrow g^{-1}(t) \leq 1$. So $\mu_{\tilde{a} \otimes \tilde{b}}(t)=\mu_{\tilde{a}}\left(g^{-1}(t)\right)=0$.

(ii) $2 \leq t \leq 9 \Rightarrow 1 \leq g^{-1}(t) \leq 3$. So $\mu_{\tilde{a} \otimes \tilde{b}}(t)=\mu_{\tilde{a}}\left(g^{-1}(t)\right)=\left(g^{-1}(t)-1\right) / 2=((2 t+3) /$ $7-1) / 2=(t-2) / 7$.

(iii) $9 \leq t \leq 30 \Rightarrow 3 \leq g^{-1}(t) \leq 6$. So $\mu_{\widetilde{a} \otimes \tilde{b}}(t)=\mu_{\widetilde{a}}\left(g^{-1}(t)\right)=1$.

(iv) $30 \leq t \leq 56 \Rightarrow 6 \leq g^{-1}(t) \leq 7$. So $\mu_{\tilde{a} \otimes \tilde{b}}(t)=\mu_{\tilde{a}}\left(g^{-1}(t)\right)=7-g^{-1}(t)=7-(t+126) /$ $26=(56-t) / 26$.

(v) $t \geq 56 \Rightarrow g^{-1}(t) \geq 7$. So $\mu_{\tilde{a} \otimes \tilde{b}}(t)=\mu_{\widetilde{a}}\left(g^{-1}(t)\right)=0$.

On the other hand, we can define $h: D_{\tilde{b}} \rightarrow D_{\widetilde{a} \otimes \tilde{b}}$ by

$$
h(t)= \begin{cases}t, & t \leq 2, \\ 7 t-12, & 2 \leq t \leq 3, \\ \frac{21 t-45}{2}, & 3 \leq t \leq 5, \\ \frac{26 t-40}{3}, & 5 \leq t \leq 8, \\ t+48, & t \geq 8\end{cases}
$$

(i) $t \leq 2 \Rightarrow h^{-1}(t) \leq 2$. So $\mu_{\widetilde{a} \otimes \tilde{b}}(t)=\mu_{\tilde{b}}\left(h^{-1}(t)\right)=0$.

(ii) $2 \leq t \leq 9 \Rightarrow 2 \leq h^{-1}(t) \leq 3$. So $\mu_{\tilde{a} \otimes \tilde{b}}(t)=\mu_{\widetilde{b}}\left(h^{-1}(t)\right)=h^{-1}(t)-2=(t+12) / 7-$ $2=(t-2) /(7)$.

(iii) $9 \leq t \leq 30 \Rightarrow 3 \leq h^{-1}(t) \leq 5$. So $\mu_{\tilde{a} \otimes \tilde{b}}(t)=\mu_{\tilde{b}}\left(h^{-1}(t)\right)=1$.

(iv) $30 \leq t \leq 56 \Rightarrow 5 \leq h^{-1}(t) \leq 8$. So $\mu_{\tilde{a} \otimes \tilde{b}}(t)=\mu_{\tilde{b}}\left(h^{-1}(t)\right)=\left(8-h^{-1}(t)\right) / 3=(8-$ $(3 t+40) / 26) / 3=(56-t) / 26$.

(v) $t \geq 56 \Rightarrow h^{-1}(t) \geq 8$. So $\mu_{\tilde{a} \otimes \tilde{b}}(t)=\mu_{\tilde{b}}\left(h^{-1}(t)\right)=0$.

From both cases, it is clear that

$$
\mu_{\tilde{a} \otimes \tilde{b}}(t)= \begin{cases}\frac{t-2}{7}, & 2 \leq t \leq 9, \\ 1, & 9 \leq t \leq 30 \\ \frac{56-t}{26}, & 30 \leq t \leq 56 \\ 0, & \text { otherwise. }\end{cases}
$$


This is the membership function of the fuzzy number $\tilde{a} \otimes \tilde{b}=\langle 2 ; 9 ; 30 ; 56\rangle$. Hence $\tilde{a} \otimes \tilde{b} \in$ $[\mu]$.

To compare this arithmetic operation with the arithmetic operation by using extension principle, we state the following example.

Example 4.3. Consider a linear triangular class $[\mu]$. Suppose $\tilde{a}=\langle 1 ; 2 ; 4\rangle, \tilde{b}=\langle 2 ; 5 ; 7\rangle \in$ $[\mu]$ are two linear triangular fuzzy numbers. The membership functions are given by

$$
\mu_{\tilde{a}}(t)=\left\{\begin{array}{ll}
t-1, & 1 \leq t \leq 2, \\
2-\frac{t}{2}, & 2 \leq t \leq 4, \\
0, & \text { otherwise, }
\end{array} \quad \mu_{\tilde{b}}(t)= \begin{cases}\frac{t-2}{3}, & 2 \leq t \leq 5 \\
\frac{7-t}{2}, & 5 \leq t \leq 7 \\
0, & \text { otherwise }\end{cases}\right.
$$

(I) The multiplication of $\tilde{a}$ and $\tilde{b}$ using the arithmetic operation as developed in Section 4. We have $\operatorname{supp}(\tilde{a} \otimes \tilde{b})=[2,28], \operatorname{core}(\tilde{a} \otimes \tilde{b})=10$, and $g: D_{\tilde{a}} \rightarrow D_{\tilde{a} * \tilde{b}}$,

$$
g(t)= \begin{cases}t+1, & t \leq 1 \\ 8 t-6, & 1 \leq t \leq 2 \\ 9 t-8, & 2 \leq t \leq 4 \\ t+24, & t \geq 4\end{cases}
$$

Now we calculate $\mu_{\tilde{a} \otimes \tilde{b}}$.

(i) $t \leq 2 \Rightarrow g^{-1}(t) \leq 1$. So $\mu_{\tilde{a} \otimes \tilde{b}}(t)=\mu_{\tilde{a}}\left(g^{-1}(t)\right)=0$.

(ii) $2 \leq t \leq 10 \Rightarrow 1 \leq g^{-1}(t) \leq 2$. So $\mu_{\tilde{a} \otimes \tilde{b}}(t)=\mu_{\widetilde{a}}\left(g^{-1}(t)\right)=g^{-1}(t)-1=(t+6) / 8-$ $1=(t-2) / 8$.

(iii) $10 \leq t \leq 28 \Rightarrow 2 \leq g^{-1}(t) \leq 4$. So $\mu_{\tilde{a} \otimes \tilde{b}}(t)=\mu_{\tilde{a}}\left(g^{-1}(t)\right)=2-g^{-1}(t) / 2=2-(t+$ $8) / 18=(28-t) / 18$.

(iv) $t \geq 28 \Rightarrow g^{-1}(t) \geq 4$. So $\mu_{\widetilde{a} \otimes \tilde{b}}(t)=\mu_{\widetilde{a}}\left(g^{-1}(t)\right)=0$.

On the other hand, we can define $h: D_{\tilde{b}} \rightarrow D_{\widetilde{a} \otimes \tilde{b}}$ by

$$
h(t)= \begin{cases}t, & t \leq 2, \\ \frac{8 t-10}{3}, & 2 \leq t \leq 5, \\ 9 t-35, & 5 \leq t \leq 7, \\ t+21, & t \geq 7\end{cases}
$$

and again calculate $\mu_{\tilde{a} \otimes \tilde{b}}$.

(i) $t \leq 2 \Rightarrow h^{-1}(t) \leq 2$. So $\mu_{\tilde{a} \otimes \tilde{b}}(t)=\mu_{\tilde{b}}\left(h^{-1}(t)\right)=0$.

(ii) $2 \leq t \leq 10 \Rightarrow 2 \leq h^{-1}(t) \leq 5$. So $\mu_{\tilde{a} \otimes \tilde{b}}(t)=\mu_{\tilde{a}}\left(h^{-1}(t)\right)=\left(h^{-1}(t)-2\right) / 3=((3 t+10) /$ $(8-2)) / 3=(t-2) / 8$. 
(iii) $10 \leq t \leq 28 \Rightarrow 5 \leq h^{-1}(t) \leq 7$. So $\mu_{\tilde{a} \otimes \tilde{b}}(t)=\mu_{\tilde{a}}\left(h^{-1}(t)\right)=\left(7-h^{-1}(t)\right) / 2=(7-(t+$ $35)) / 9 / 2=(28-t) / 18$.

(iv) $t \geq 28 \Rightarrow h^{-1}(t) \geq 4$. So $\mu_{\tilde{a} \otimes \tilde{b}}(t)=\mu_{\tilde{a}}\left(h^{-1}(t)\right)=0$.

Thus from both cases, it is clear that

$$
\mu_{\tilde{a} \otimes \tilde{b}}(t)= \begin{cases}\frac{t-2}{8}, & 2 \leq t \leq 10, \\ \frac{28-t}{18}, & 10 \leq t \leq 28 \\ 0, & \text { otherwise. }\end{cases}
$$

This is the membership function of the linear triangular fuzzy number $\tilde{a} \otimes \tilde{b}=\langle 2 ; 10 ; 28\rangle$.

(II) The multiplication of $\tilde{a}$ and $\tilde{b}$ using the extension principle.

By the extension principle of the arithmetic operations of fuzzy numbers, the multiplication of the two fuzzy numbers $\tilde{a}$ and $\tilde{b}$ with linear triangular membership functions as given in Example 4.3 would have been the following fuzzy number $\tilde{a} \otimes \tilde{b}$ say, whose membership function is given by

$$
\mu_{\tilde{a} \otimes \tilde{b}}(t)= \begin{cases}\frac{1}{6}(\sqrt{12 t+1}-5), & 2 \leq t \leq 10, \\ \frac{1}{4}(11-\sqrt{4 t+9}), & 10 \leq t \leq 28, \\ 0, & \text { otherwise. }\end{cases}
$$

Here $\mu_{\tilde{a} \otimes \tilde{b}}$ is a nonlinear membership function even though the two given fuzzy numbers were linear triangular. That is, the multiplication of two linear triangular fuzzy numbers is not a linear triangular fuzzy number by the usual fuzzy multiplication. But in (I), it has been verified that multiplication of two linear triangular fuzzy numbers is a linear triangular fuzzy number.

Scalar multiplication. The scalar multiplication between a crisp number $\alpha$ and a fuzzy number $\tilde{a}=\left\langle l_{a} ; a_{*} ; a^{*} ; r_{a}\right\rangle$ with $\mu_{\tilde{a}}$ as membership function is done as follows.

(i) If $\alpha \geq 0$, then $\alpha \tilde{a}=\left\langle\alpha l_{a} ; \alpha a_{*} ; \alpha a^{*} ; \alpha r_{a}\right\rangle$, here a map $f: D_{\tilde{a}} \rightarrow D_{\alpha \tilde{a}}$ can be defined as in Theorem 4.1 and the membership function is given by $\mu_{\alpha \tilde{a}}=\mu_{\tilde{a}}\left(f^{-1}(t)\right)$.

(ii) If $\alpha<0$, then $\alpha \tilde{a}=\left\langle\alpha r_{a} ; \alpha a^{*} ; \alpha a_{*} ; \alpha l_{a}\right\rangle$ here again a map $f: D_{\tilde{a}} \rightarrow D_{\alpha \tilde{a}}$ can be defined as in Theorem 4.1 and the membership function is given by $\mu_{\alpha \tilde{a}}=$ $\mu_{\tilde{a}}\left(f^{-1}(t)\right)$.

Example 4.4. Consider $\tilde{a}=\langle 1 ; 3 ; 6 ; 7\rangle, \tilde{b}=\langle 2 ; 3 ; 5 ; 8\rangle$ as in Example 4.2 and $\alpha=2, \beta=-3$. Then $2 \tilde{a}=\langle 2 ; 6 ; 12 ; 14\rangle,-3 \tilde{a}=\langle-21 ;-18 ;-9 ;-3\rangle$. 
Take $\tilde{a}=\langle 1 ; 2 ; 4\rangle$ as in Example 4.3 and $\alpha=2, \beta=-3$. Then $2 \tilde{a}=\langle 2 ; 4 ; 8\rangle,-3 \tilde{a}=$ $\langle-12 ;-6 ;-3\rangle$, the corresponding membership functions are given below:

$$
\mu_{2 \widetilde{a}}(t)=\left\{\begin{array}{ll}
\frac{t-2}{2}, & 2 \leq t \leq 4, \\
\frac{8-t}{4}, & 4 \leq t \leq 8, \\
0, & \text { otherwise }
\end{array} \quad \mu_{-3 \tilde{a}}(t)= \begin{cases}t+12, & -12 \leq t \leq-6, \\
\frac{-3-t}{3}, & -6 \leq t \leq-3, \\
0, & \text { otherwise. }\end{cases}\right.
$$

With the above knowledge of equivalence class in the set of fuzzy numbers and the arithmetic operations in a class, we introduce $[\mu]$-fuzzy matrix in the next section.

\section{5. $[\mu]$-fuzzy matrix}

A $[\mu]$-fuzzy matrix of order $m \times n$ is an arrangement of $m n$ fuzzy numbers of class $[\mu]$ in $m$ rows and $n$ columns. It is denoted by $\tilde{A}_{\mu}$, where $\tilde{A}_{\mu}=\left(\tilde{a}_{i j}\right)_{m \times n}$, where $\tilde{a}_{i j} \in[\mu]$.

Two fuzzy matrices of same class and same order can be added and two fuzzy matrices of order $m \times n$ and $n \times p$, respectively, in the same class can be multiplied in the sense of arithmetic operation of fuzzy numbers in an equivalence class as defined in Section 4. The arithmetic operations such as addition, multiplication of two $[\mu]$-fuzzy matrices, and the multiplication of a $[\mu]$-fuzzy matrix with a scalar are defined as follows.

(1) Addition: for the fuzzy matrices $\widetilde{A}_{\mu}=\left(\tilde{a}_{i j}\right)$ and $\widetilde{B}_{\mu}=\left(\tilde{b}_{i j}\right)$ of order $m \times n, \widetilde{A}_{\mu} \oplus \widetilde{B}_{\mu}=$ $\left(\tilde{a}_{i j} \oplus \tilde{b}_{i j}\right)$.

(2) Multiplication: for the fuzzy matrices $\widetilde{A}_{\mu}=\left(\tilde{a}_{i j}\right)$ of order $m \times n$ and $\widetilde{B}_{\mu}=\left(\tilde{b}_{i j}\right)$ of order $n \times p, \widetilde{A}_{\mu} \otimes \widetilde{B}_{\mu}=\left(\widetilde{c}_{r s}\right)$ is of order $m \times p$, where $\tilde{c}_{r s}=\left(\tilde{a}_{r 1} \otimes \tilde{b}_{1 s}\right) \oplus\left(\tilde{a}_{r 2} \otimes\right.$ $\left.\tilde{b}_{2 s}\right) \oplus \cdots \oplus\left(\tilde{a}_{r n} \otimes \tilde{b}_{n s}\right)$.

(3) Scalar multiplication: for a scalar $\alpha, \alpha \tilde{A}_{\mu}=\left(\alpha \tilde{a}_{i j}\right)$,

where $\oplus$ is the addition of two fuzzy numbers, $\otimes$ is the multiplication of two fuzzy numbers. $\oplus$ and $\otimes$ can be evaluated as the general arithmetic operation $*$ defined in Section 4 .

Example 5.1. Let

$$
\widetilde{A}_{\mu}=\left[\begin{array}{ll}
\tilde{2} & \tilde{1} \\
\tilde{2} & \tilde{3}
\end{array}\right], \quad \widetilde{B}_{\mu}=\left[\begin{array}{ll}
\tilde{1} & \tilde{1} \\
\tilde{2} & \tilde{2}
\end{array}\right]
$$

where $\tilde{2}=\langle 1 ; 2 ; 4\rangle, \widetilde{1}=\langle 0 ; 1 ; 2\rangle, \widetilde{3}=\langle 1 ; 3 ; 4\rangle, \tilde{2}, \tilde{1}, \tilde{3} \in[\mu]$, where $[\mu]$ is the linear triangular class fuzzy numbers

$$
\widetilde{A}_{\mu} \bigoplus \widetilde{B}_{\mu}=\left[\begin{array}{cc}
\tilde{3} & \tilde{2} \\
\tilde{4} & \tilde{5}
\end{array}\right], \quad \widetilde{A}_{\mu} \bigotimes \widetilde{B}_{\mu}=\left[\begin{array}{cc}
\tilde{4} & \tilde{4} \\
\tilde{8} & \tilde{8}
\end{array}\right], \quad 2 \cdot \widetilde{A}_{\mu}=\left[\begin{array}{cc}
\tilde{4} & \tilde{2} \\
\tilde{4} & \tilde{6}
\end{array}\right]
$$

where in $\widetilde{A}_{\mu} \oplus \widetilde{B}_{\mu}, \widetilde{3}=\langle 1 ; 3 ; 6\rangle, \tilde{2}=\langle 0 ; 2 ; 4\rangle, \tilde{4}=\langle 2 ; 4 ; 8\rangle, \tilde{5}=\langle 2 ; 5 ; 8\rangle$, in $\widetilde{A}_{\mu} \otimes \widetilde{B}_{\mu}, \tilde{4}=$ $\langle 0 ; 4 ; 16\rangle, \widetilde{8}=\langle 1 ; 8 ; 24\rangle$, and in $2 \cdot \widetilde{A}_{\mu}, \widetilde{4}=\langle 2 ; 4 ; 8\rangle, \widetilde{6}=\langle 2 ; 6 ; 8\rangle$. 
Note that $\tilde{4}=\langle 0 ; 4 ; 16\rangle, \tilde{4}=\langle 2 ; 4 ; 8\rangle$ are two different fuzzy numbers since $I_{\lambda}(\langle 0 ; 4 ; 16\rangle) \neq$ $I_{\lambda}(\langle 2 ; 4 ; 8\rangle)$. But they are similar and are in same linear triangular class.

Definition 5.2 (symmetric fuzzy matrix). A $[\mu]$-fuzzy matrix $\widetilde{A}_{\mu}=\left(\tilde{a}_{i j}\right)$ of order $n \times n$ is said to be symmetric if $\mu_{\tilde{a}_{i j}}(t)=\mu_{\tilde{a}_{j i}}(t)$ for all $i \neq j$ and $t \in \mathbb{R}$.

In the literature, a lot of defuzzification methods or ranking of fuzzy numbers are available. Here we have considered the ranking method applied through $\lambda$-integral value to define a positive semidefinite fuzzy matrix. However, any other ranking method can be applied.

Definition 5.3. Positive semidefinite fuzzy matrix. A $[\mu]$-fuzzy matrix $\widetilde{A}_{\mu}$ of order $n \times n$ is said to be positive semidefinite if for $x \in \mathbb{R}^{n}, I_{\lambda}\left(x^{T} \tilde{A}_{\mu} x\right) \geq 0$.

Example 5.4. The following matrix is a symmetric fuzzy matrix of order $3 \times 3$.

Let

$$
\widetilde{A_{\mu}}=\left[\begin{array}{ccc}
\tilde{0} & \tilde{2} & \tilde{4} \\
\tilde{2} & \tilde{3} & \tilde{4} \\
\tilde{4} & \tilde{4} & \tilde{2}
\end{array}\right],
$$

where $\tilde{a}_{i j} \in[\mu], \mu$ is the membership function of linear triangular fuzzy numbers in $F$. Specifically, $\tilde{a}_{11}=\tilde{0}=\langle-1 ; 0 ; 2\rangle, \tilde{a}_{12}=\tilde{2}=\langle 1 ; 2 ; 4\rangle, \tilde{a}_{13}=\tilde{a}_{31}=\tilde{4}=\langle 0 ; 4 ; 5\rangle, \tilde{a}_{22}=\tilde{3}=$ $\langle 0 ; 3 ; 7\rangle, \tilde{a}_{23}=\tilde{a}_{32}=\tilde{4}=\langle 1 ; 4 ; 8\rangle$, and $\tilde{a}_{33}=\tilde{2}=\langle 1 ; 2 ; 3\rangle$.

Example 5.5. The following $[\mu]$-matrix is positive semidefinite.

Let $\widetilde{A}_{\mu}=\left[\begin{array}{cc}\tilde{6} & \tilde{4} \\ \widetilde{4} & \frac{10}{10}\end{array}\right]$, where $[\mu]$ is the linear triangular class, $\widetilde{6}=\langle 4 ; 6 ; 7\rangle, \widetilde{4}=\langle 3 ; 4 ; 5\rangle, \widetilde{10}=$ $\langle 9 ; 10 ; 12\rangle$, and $x=\left[x_{1}, x_{2}\right], x_{1}, x_{2} \in \mathbb{R}$.

If either $x_{1}<0$ or $x_{2}<0$ but not both, then

$$
x^{T} \tilde{A}_{\mu} x=\left\langle 4 x_{1}^{2}+10 x_{1} x_{2}+9 x_{2}^{2} ; 6 x_{1}^{2}+8 x_{1} x_{2}+10 x_{2}^{2} ; 7 x_{1}^{2}+6 x_{1} x_{2}+12 x_{2}^{2}\right\rangle .
$$

Here we can see that

$$
\begin{gathered}
4 x_{1}^{2}+10 x_{1} x_{2}+9 x_{2}^{2}=\left(2 x_{1}+3 x_{2}\right)^{2}-2 x_{1} x_{2}>0 \\
6 x_{1}^{2}+8 x_{1} x_{2}+10 x_{2}^{2}=\left(\sqrt{6} x_{1}+\sqrt{10} x_{2}\right)^{2}+(8-2 \sqrt{60}) x_{1} x_{2}>0 \\
7 x_{1}^{2}+6 x_{1} x_{2}+12 x_{2}^{2}=\left(\sqrt{7} x_{1}+\sqrt{12} x_{2}\right)^{2}+(6-2 \sqrt{84}) x_{1} x_{2}>0 .
\end{gathered}
$$

Otherwise, when both $x_{1}$ and $x_{2}$ are either $>0$ or $<0$, then

$$
x^{T} \tilde{A}_{\mu} x=\left\langle 4 x_{1}^{2}+6 x_{1} x_{2}+9 x_{2}^{2} ; 6 x_{1}^{2}+8 x_{1} x_{2}+10 x_{2}^{2} ; 7 x_{1}^{2}+10 x_{1} x_{2}+12 x_{2}^{2}\right\rangle .
$$

In this case, it is clear that all the expressions are positive. 


\section{Equivalence class in fuzzy numbers}

Hence $I_{\lambda}\left(x^{T} \tilde{A}_{\mu} x\right)>0$ for all $x \in \mathbb{R}$.

Therefore, $\tilde{A}_{\mu}$ is positive semidefinite.

Definition 5.6 ([ $\mu]$-crisp matrix). Every $[\mu]$-fuzzy matrix, $\tilde{A}_{\mu}$, is associated with a pair of crisp matrices $A_{\mu}^{-}(\theta)$ and $A_{\mu}^{+}(\theta)$ for each $0 \leq \theta<1$. Define these as

$$
\begin{aligned}
& A_{\mu}^{-}(\theta)=\left(\theta_{i j}^{-}\right), \quad \text { where } \theta_{i j}^{-}=\mu_{\tilde{a}_{i j} L}^{-1}(\theta), \\
& A_{\mu}^{+}(\theta)=\left(\theta_{i j}^{+}\right), \quad \text { where } \theta_{i j}^{+}=\mu_{\tilde{a}_{i j} R}^{-1}(\theta) .
\end{aligned}
$$

Example 5.7. The $[\mu]$-fuzzy matrix in Example 5.5, $\widetilde{A}_{\mu}=\left[\begin{array}{cc}\tilde{6} & \widetilde{4} \\ \widetilde{4} & \frac{10}{10}\end{array}\right]$, is associated with two crisp matrices

$$
\begin{aligned}
& A_{\mu}^{-}(0.1)=\left[\begin{array}{ll}
4.2 & 3.1 \\
3.1 & 9.1
\end{array}\right], \\
& A_{\mu}^{+}(0.1)=\left[\begin{array}{cc}
6.9 & 4.9 \\
4.9 & 11.8
\end{array}\right]
\end{aligned}
$$

for $\theta=0.1$.

Proposition 5.8. If a $[\mu]$-fuzzy matrix is symmetric, then the associated crisp matrices are also symmetric.

Proof. The proof is easy and we omitted it.

Next section and its subsection are devoted to the application of $[\mu]$-class and $[\mu]$ fuzzy matrix in decision-making problems.

\section{Fuzzy decision-making problem}

The general form of a fuzzy decision-making problem is

$$
\begin{gathered}
\operatorname{minimize} \tilde{f}(x), \\
\text { subject to } \tilde{g}(x) \preceq(\succeq, \simeq) \widetilde{0}, \\
x \geq 0,
\end{gathered}
$$

where $\tilde{f}(x), \tilde{g}(x)$ are fuzzy functions from $\mathbb{R}^{+}$to the set of fuzzy numbers.

A lot of defuzzification methods have been developed so far to solve such type of fuzzy decision-making problems. In this section, we have presented the following algorithm to solve the problem (6.1) without defuzzifying and using the concept of fuzzy equivalence class as developed in previous sections.

Suppose that the coefficients of $\tilde{f}(x), \tilde{g}(x)$ are fuzzy numbers belonging to $[\mu]$-class and $\tilde{0} \in[\mu]$. 
Step 1. For a particular aspiration level $\theta \in[0,1]$, problem (6.1) can be decomposed to the following two decision-making problems using $[\mu]$-crisp matrix concept:

$$
\begin{gathered}
\text { minimize } \tilde{f}_{\mu}^{-}(\theta, x), \\
\text { subject to } \tilde{g}_{\mu}^{-}(\theta, x) \leq(=, \geq) \tilde{0}_{\mu}^{-}(\theta), \\
0 \leq \theta \leq 1, \\
x \geq 0, \\
\operatorname{minimize} \tilde{f}_{\mu}^{+}(\theta, x), \\
\text { subject to } \tilde{g}_{\mu}^{+}(\theta, x) \leq(=, \geq) \widetilde{0}_{\mu}^{+}(\theta), \\
0 \leq \theta \leq 1, \\
x \geq 0 .
\end{gathered}
$$

For a particular aspiration level $\theta \in(0,1)$, we use (6.2) to get the solution of (6.1).

Step 2. Formulate the corresponding multiobjective problem:

$$
\begin{aligned}
& \text { minimize }\left\{\tilde{f}_{\mu}^{-}(\theta, x), \tilde{f}_{\mu}^{+}(\theta, x)\right\}, \\
& \text { subject to } \tilde{g}_{\mu}^{-}(\theta, x) \leq(=, \geq) \tilde{0}_{\mu}^{-}(\theta), \\
& \tilde{g}_{\mu}^{+}(\theta, x) \leq(=, \geq) \tilde{0}_{\mu}^{+}(\theta), \\
& 0 \leq \theta \leq 1, \\
& x \geq 0 .
\end{aligned}
$$

Step 3. The multiobjective problem (6.3) can be solved for each aspiration level by any multiobjective programming method.

This algorithm can be explained through the following fuzzy quadratic programming problem.

6.1. Fuzzy quadratic programming. The general form of a crisp quadratic programming problem is

$$
\begin{gathered}
\operatorname{minimize} x^{T} A x+c^{T} x, \\
\text { subject to } B x \leq(\geq,=) b, \\
x \geq 0,
\end{gathered}
$$

where $x \in \mathbb{R}^{n}, A$ is a positive semidefinite symmetric crisp matrix of order $n \times n, B, c$ and $b$ are crisp matrices of order $m \times n, n \times 1$, and $m \times 1$, respectively.

We define a fuzzy quadratic programming problem as follows:

$$
\begin{gathered}
\operatorname{minimize} x^{T} \widetilde{A}_{\mu} x \oplus \widetilde{c}_{\mu}^{T} x, \\
\text { subject to } \widetilde{B}_{\mu} x \preceq(\succeq, \simeq) \widetilde{b}_{\mu}, \\
x \geq 0,
\end{gathered}
$$


where $x \in \mathbb{R}^{n}, \tilde{A}_{\mu}$ is a positive semidefinite symmetric $[\mu]$-fuzzy matrix of order $n \times n$, $\widetilde{B}_{\mu}, \widetilde{c}_{\mu}$, and $\tilde{b}_{\mu}$ are $[\mu]$-fuzzy matrices of order $m \times n, n \times 1$, and $m \times 1$, respectively. Let $\tilde{f}(x)=x^{T} \tilde{A}_{\mu} x \oplus \tilde{c}^{T} x$ and $g(\widetilde{x})=\widetilde{B}_{\mu} x \oplus \tilde{d}_{\mu}$, where $\tilde{d}_{\mu}=(-1) \tilde{b}_{\mu}$.

Let

$$
\tilde{A}_{\mu}=\left[\begin{array}{cc}
\tilde{6} & \tilde{4} \\
\tilde{4} & \widetilde{10}
\end{array}\right], \quad \tilde{c}_{\mu}=\left[\begin{array}{c}
\tilde{1} \\
-\tilde{2}
\end{array}\right], \quad \widetilde{B}_{\mu}=\left[\begin{array}{cc}
\tilde{2} & \tilde{1} \\
\tilde{1} & \tilde{1}
\end{array}\right], \quad \tilde{b}_{\mu}=\left[\begin{array}{c}
\tilde{1} \\
\tilde{4}
\end{array}\right],
$$

where $[\mu]$ is the linear triangular class, $\tilde{1}=\langle 0 ; 1 ; 3\rangle, \tilde{2}=\langle 1 ; 2 ; 4\rangle, \tilde{4}=\langle 3 ; 4 ; 5\rangle, \tilde{6}=\langle 4 ; 6 ; 7\rangle$, $\widetilde{10}=\langle 9 ; 10 ; 12\rangle$. So $-\widetilde{2}=\langle-4 ;-2 ;-1\rangle, x=\left(x_{1}, x_{2}\right)^{T}$. In Example 5.5, it is verified that $I_{\lambda}\left(x^{T} \widetilde{A}_{\mu} x\right) \geq 0$ for all $x \in \mathbb{R}^{2}$ and some $0 \leq \lambda \leq 1$. Hence $\tilde{A}_{\mu}$ is a positive semidefinite $[\mu]$-fuzzy matrix. Also $\widetilde{A}_{\mu}$ is symmetric. We consider the fuzzy quadratic programming problem (6.5) as

$$
\begin{aligned}
& \min \tilde{6} x_{1}^{2} \oplus(\tilde{4} \oplus \tilde{4}) x_{1} x_{2} \oplus \widetilde{10} x_{2}^{2} \oplus \tilde{1} x_{1} \oplus(-1) \tilde{2} x_{2}, \\
& \text { subject to } \tilde{2} x_{1} \oplus \tilde{1} x_{2} \succeq \tilde{1}, \\
& \tilde{1} x_{1} \oplus \tilde{1} x_{2} \preceq \tilde{4}, \\
& x_{1}, x_{2} \geq 0 .
\end{aligned}
$$

Solution using the algorithm. For some aspiration level $\theta$, each of $\widetilde{A}_{\mu}, \widetilde{c}_{\mu}^{T}, \tilde{b}_{\mu}, \widetilde{B}_{\mu}$ corresponds to a pair of crisp matrices $\left(A_{\mu}^{-}(\theta), A_{\mu}^{+}(\theta)\right),\left(c_{\mu}^{-}(\theta), c_{\mu}^{+}(\theta)\right),\left(b_{\mu}^{-}(\theta), b_{\mu}^{+}(\theta)\right),\left(B_{\mu}^{-}(\theta)\right.$, $\left.B_{\mu}^{+}(\theta)\right)$.

Hence the fuzzy quadratic programming problem (6.5) corresponds to a pair of crisp quadratic programming problems

$$
\begin{gathered}
\min x^{T} A_{\mu}^{-}(\theta) x+c_{\mu}^{-T}(\theta) x, \\
\text { subject to } B_{\mu}^{-}(\theta) x \leq(=, \geq) b_{\mu}^{-}(\theta), \\
x \geq 0, \\
\text { minimize } x^{T} A_{\mu}^{+}(\theta) x+c_{\mu}^{+T}(\theta) x, \\
\text { subject to } B_{\mu}^{+}(\theta) x \leq(=, \geq) b_{\mu}^{+}(\theta), \\
x \geq 0,
\end{gathered}
$$

where

$$
\begin{gathered}
A_{\mu}^{-}(\theta)=\left[\begin{array}{cc}
2 \theta+4 & \theta+3 \\
\theta+3 & 9+\theta
\end{array}\right], \quad A_{\mu}^{+}(\theta)=\left[\begin{array}{cc}
7-\theta & 5-\theta \\
5-\theta & 12-2 \theta
\end{array}\right], \quad c_{\mu}^{-}(\theta)=\left[\begin{array}{c}
\theta \\
2 \theta-4
\end{array}\right], \\
c_{\mu}^{+}(\theta)=\left[\begin{array}{c}
3-2 \theta \\
-1-\theta
\end{array}\right], \quad B_{\mu}^{-}(\theta)=\left[\begin{array}{cc}
\theta+1 & \theta \\
\theta & \theta
\end{array}\right], \quad B_{\mu}^{+}(\theta)=\left[\begin{array}{cc}
4-2 \theta & 3-2 \theta \\
3-2 \theta & 3-2 \theta
\end{array}\right], \\
b_{\mu}^{-}(\theta)=\left[\begin{array}{c}
\theta \\
\theta+3
\end{array}\right], \quad b_{\mu}^{+}(\theta)=\left[\begin{array}{c}
3-2 \theta \\
5-\theta
\end{array}\right] .
\end{gathered}
$$


Step 1. Substituting the values of the pair of crisp matrices, (6.7) can be converted to two crisp quadratic programming problems in the light of (6.8) as

$$
\begin{aligned}
& \operatorname{minimize}(2 \theta+4) x_{1}^{2}+2(\theta+3) x_{1} x_{2}+(9+\theta) x_{2}^{2}+\theta x_{1}+(2 \theta-4) x_{2}, \\
& \text { subject to }(\theta+1) x_{1}+\theta x_{2} \geq \theta, \\
& \qquad\left(x_{1}+x_{2}\right) \leq \theta+3, \\
& \quad x_{1} \geq 0, \quad x_{2} \geq 0, \quad 0 \leq \theta \leq 1,
\end{aligned}
$$

minimize $(7-\theta) x_{1}^{2}+2(5-\theta) x_{1} x_{2}+(12-2 \theta) x_{2}^{2}+(3-2 \theta) x_{1}+(-1-\theta) x_{2}$,

subject to $(4-2 \theta) x_{1}+(3-2 \theta) x_{2} \geq 3-2 \theta$,

$$
\begin{aligned}
& (3-2 \theta)\left(x_{1}+x_{2}\right) \leq 5-\theta, \\
& x_{1} \geq 0, \quad x_{2} \geq 0, \quad 0 \leq \theta \leq 1 .
\end{aligned}
$$

Step 2. The corresponding multiobjective programming is

$$
\begin{aligned}
& \min \left\{(2 \theta+4) x_{1}^{2}+2(\theta+3) x_{1} x_{2}+(9+\theta) x_{2}^{2}+\theta x_{1}+(2 \theta-4) x_{2},\right. \\
& \left.\quad(7-\theta) x_{1}^{2}+2(5-\theta) x_{1} x_{2}+(12-2 \theta) x_{2}^{2}+(3-2 \theta) x_{1}+(-1-\theta) x_{2}\right\} \\
& \text { subject to }(\theta+1) x_{1}+\theta x_{2} \geq \theta, \quad \theta\left(x_{1}+x_{2}\right) \leq \theta+3 \\
& \quad(4-2 \theta) x_{1}+(3-2 \theta) x_{2} \geq 3-2 \theta \\
& \quad(3-2 \theta)\left(x_{1}+x_{2}\right) \leq 5-\theta \\
& \quad x_{1} \geq 0, \quad x_{2} \geq 0, \quad 0 \leq \theta \leq 1
\end{aligned}
$$

Step 3. Using weighted average method, the above multiobjective programming becomes

$$
\begin{gathered}
\min \lambda\left[(2 \theta+4) x_{1}^{2}+2(\theta+3) x_{1} x_{2}+(9+\theta) x_{2}^{2}+\theta x_{1}+(2 \theta-4) x_{2}\right] \\
+(1-\lambda)\left[(7-\theta) x_{1}^{2}+2(5-\theta) x_{1} x_{2}+(12-2 \theta) x_{2}^{2}\right. \\
\left.+(3-2 \theta) x_{1}+(-1-\theta) x_{2}\right], \\
\text { subject to }(\theta+1) x_{1}+\theta x_{2} \geq \theta, \quad \theta\left(x_{1}+x_{2}\right) \leq \theta+3, \\
\\
(4-2 \theta) x_{1}+(3-2 \theta) x_{2} \geq 3-2 \theta, \\
\\
(3-2 \theta)\left(x_{1}+x_{2}\right) \leq 5-\theta, \\
x_{1} \geq 0, \quad x_{2} \geq 0, \quad 0 \leq \theta \leq 1 .
\end{gathered}
$$

Solution of this problem is tabulated for each value of $\theta$ by considering $\lambda=0.3$ in Table 6.1.

Here the solution is derived using the concept of fuzzy equivalence class and $[\mu]$-fuzzy matrix. The fuzzy quadratic problem is solved for 11 aspiration levels and summarized in the table. Each problem is solved by Lingo package. The advantage of this method, compared to any defuzzification method, is that the solution corresponds to a particular aspiration level. 
Table 6.1

\begin{tabular}{lccc}
\hline$\theta$ & $x_{1}$ & $x_{2}$ & $f(x)$ \\
\hline 0 & 0.5732861 & 0.2356186 & 4.565938 \\
0.1 & 0.5700244 & 0.2263955 & 4.369359 \\
0.2 & 0.5659454 & 0.2163832 & 4.163395 \\
0.3 & 0.5608270 & 0.2054951 & 3.946557 \\
0.4 & 0.5543727 & 0.1936398 & 3.717039 \\
0.5 & 0.5461818 & 0.1807273 & 3.472718 \\
0.6 & 0.5357056 & 0.1666801 & 3.211037 \\
0.7 & 0.5221812 & 0.1514556 & 2.928989 \\
0.8 & 0.5045319 & 0.1350882 & 2.623118 \\
0.9 & 0.4812167 & 0.1177694 & 2.289659 \\
1.0 & 0.4500000 & 0.1000000 & 1.925000 \\
\hline
\end{tabular}

\section{Conclusion}

In this paper, the objective of introducing fuzzy equivalence class is to find the membership value of arithmetic operations of a large number of same type of fuzzy numbers and to apply this in fuzzy decision-making problems. The introduction of $[\mu]$-fuzzy matrix is to formulate fuzzy quadratic programming in which the matrix involved in the quadratic form of the objective function has to be symmetric positive semidefinite. Also this paper deals with a solution method for fuzzy decision-making problem. The basic concept of this method is to decompose the original fuzzy decision-making problem into two problems for a particular aspiration level. This methodology is applicable whenever all the fuzzy numbers involved in the decision-making problem are of same type belonging to one equivalence class. If the fuzzy numbers are of different type, that is, belonging to different equivalent classes, then such type of decomposition of the fuzzy decision-making problem for an aspiration level will be difficult since in that case, the arithmetic operation of fuzzy numbers is not closed. This difficulty can be solved if a one-to-one correspondence between two equivalence classes can be established. This is the future research scope of this paper.

\section{Acknowledgments}

The authors would like to thank the referees for their valuable suggestions which improved the presentation of the paper. The first author is thankful to ISIRD, IIT Kharagpur for financial support to prepare this paper. Research work of the second author is supported by AICTE, India, and G. H. Patel College of Engineering and Technology, V. V. Nagar-388120, Gujarat, India, and IIT Kharagpur.

\section{References}

[1] R. E. Bellman and L. A. Zadeh, Decision-making in a fuzzy environment, Management Science 17 (1970-1971), B141-B164.

[2] D. Dubois and H. Prade, Operations on fuzzy numbers, International Journal of Systems Science 9 (1978), no. 6, 613-626. 
[3] T.-S. Liou and M. J. J. Wang, Ranking fuzzy numbers with integral value, Fuzzy Sets and Systems 50 (1992), no. 3, 247-255.

[4] M. Mizumoto and K. Tanaka, Algebraic properties of fuzzy numbers, Proceedings of the IEEE International Conference on Cybernetics and Society, Washington, DC, 1976.

[5] R. E. Moore, Interval Analysis, Prentice-Hall, New Jersey, 1966.

[6] B. Werners, An interactive fuzzy programming system, Fuzzy Sets and Systems 23 (1987), no. 1, 131-147.

[7] L. A. Zadeh, The concept of a linguistic variable and its application to approximate reasoning. I, Information Science 8 (1975), no. 3, 199-249.

[8] _ The concept of a linguistic variable and its application to approximate reasoning. II, Information Science 8 (1975), no. 4, 301-357.

[9] H.-J. Zimmermann, Fuzzy mathematical programming, Computers \& Operations Research and Their Application to Problems of World Concern 10 (1983), no. 4, 291-298.

Geetanjali Panda: Department of Mathematics, Indian Institute of Technology, Kharagpur-721302, West Bengal, India

E-mail address: geetanjali@maths.iitkgp.ernet.in

Motilal Panigrahi: Department of Mathematics, Indian Institute of Technology, Kharagpur-721302, West Bengal, India

E-mail address: motilal.panigrahi@gmail.com

Sudarsan Nanda: Department of Mathematics, Indian Institute of Technology, Kharagpur-721302, West Bengal, India

E-mail address: snanda@maths.iitkgp.ernet.in 


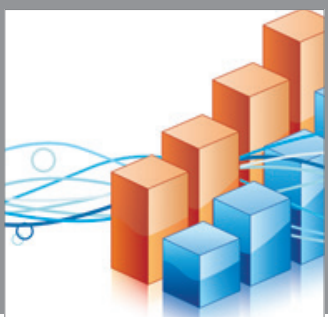

Advances in

Operations Research

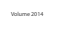

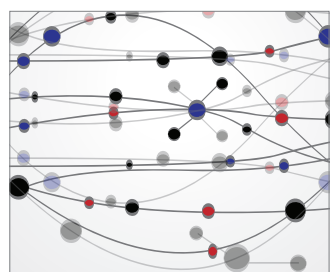

\section{The Scientific} World Journal
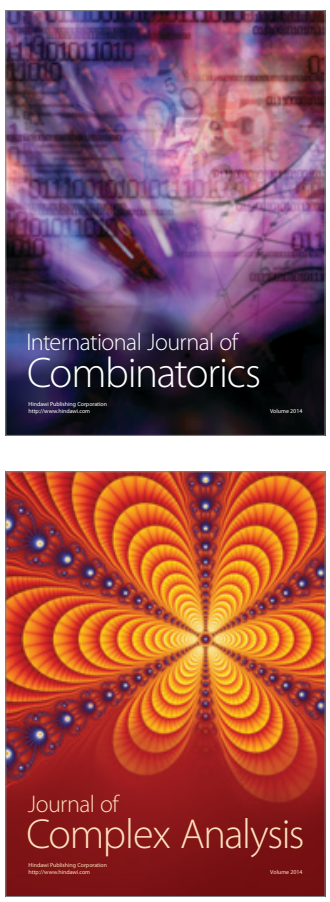

International Journal of

Mathematics and

Mathematical

Sciences
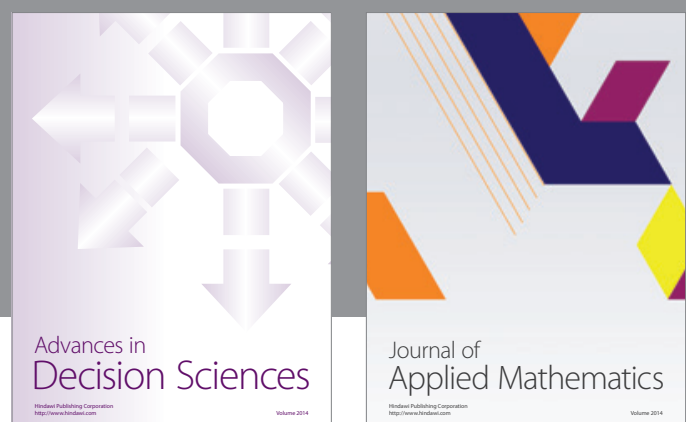

Journal of

Applied Mathematics
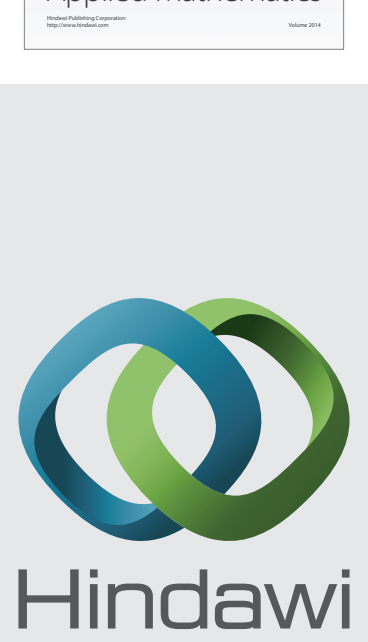

Submit your manuscripts at http://www.hindawi.com
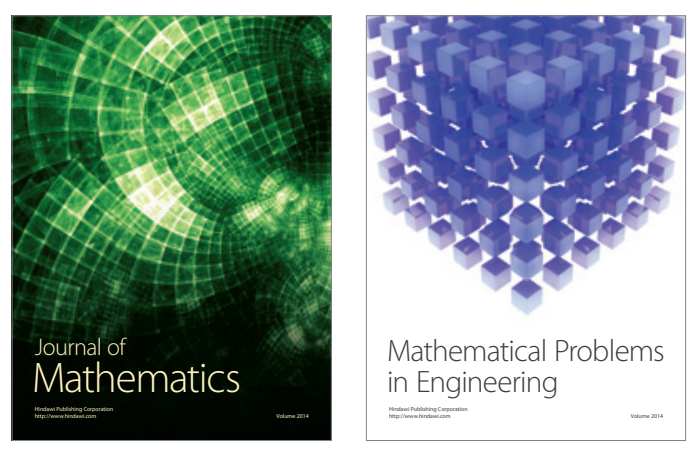

Mathematical Problems in Engineering
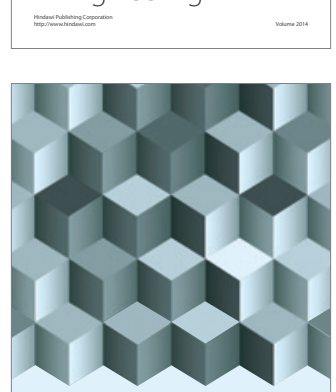

Journal of

Function Spaces
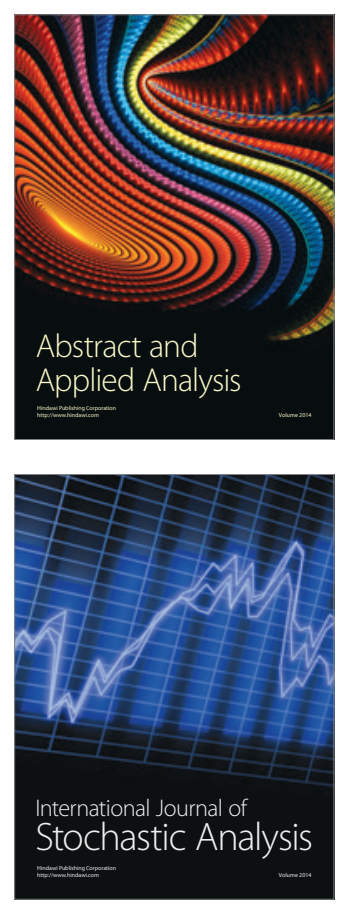

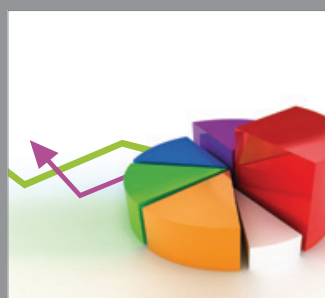

ournal of

Probability and Statistics

Promensencen
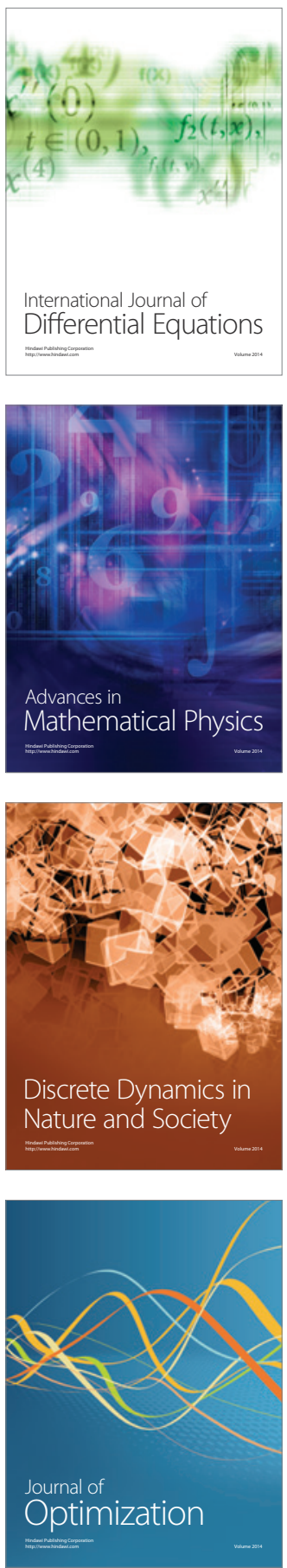